ALEA, Lat. Am. J. Probab. Math. Stat. 17, 759-773 (2020)

DOI: $10.30757 /$ ALEA.v17-29

\title{
A CLT for the total energy of the two-dimensional critical Ising model
}

\section{Jianping Jiang}

NYU-ECNU Institute of Mathematical Sciences at NYU Shanghai, 3663 Zhongshan Road North, Shanghai 200062, China.

E-mail address: jjiang@nyu.edu

Abstract. Consider the Ising model on $([1,2 N] \times[1,2 M]) \cap \mathbb{Z}^{2}$ at critical temperature with periodic boundary condition in the horizontal direction and free boundary condition in the vertical direction. Let $E_{M, N}$ be its total energy (or Hamiltonian). Suppose $M$ is a function of $N$ satisfying $M \geq N /(\ln N)^{\alpha}$ for some $\alpha \in[0,1)$. In particular, one may take $M=N$. We prove that

$$
\frac{E_{M, N}+4 \sqrt{2} M N-(4 / \pi) N \ln N}{\sqrt{(32 / \pi) M N \ln N}}
$$

converges weakly to a standard Gaussian distribution as $N \rightarrow \infty$.

\section{Introduction}

Recall that the classical Ising model at inverse temperature $\beta$ on $\Lambda \subset \mathbb{Z}^{d}$ with free boundary condition is defined by the probability measure $\mathbb{P}_{\Lambda}$ on $\{-1,+1\}^{\Lambda}$ such that for each $\sigma \in\{-1,+1\}^{\Lambda}$,

$$
\mathbb{P}_{\Lambda}(\sigma):=\frac{1}{Z_{\Lambda}(\beta)} e^{\beta \sum_{\{i, j\}} \sigma_{i} \sigma_{j}},
$$

where the sum is over all nearest neighbor pairs in $\Lambda$, and $Z_{\Lambda}(\beta)$ is the partition function (which is the normalization constant needed to make this a probability measure). The total magnetization and total energy (or Hamiltonian) are

$$
\begin{aligned}
& M_{\Lambda}:=\sum_{i \in \Lambda} \sigma_{i}, \\
& E_{\Lambda}:=-\sum_{\{i, j\}} \sigma_{i} \sigma_{j} .
\end{aligned}
$$

Received by the editors September 9h, 2019; accepted August 1st, 2020.

2010 Mathematics Subject Classification. Primary: 82B20, 82B27; Secondary: 82B23, 60F05.

Key words and phrases. Ising model; total energy; critical; central limit theorem. 
It was proved in Newman (1983) that under the full-plane Ising measure $\mathbb{P}$ (with the corresponding expectation $\mathbb{E}),\left(\left(M_{\Lambda}-\mathbb{E} M_{\Lambda}\right) / \operatorname{Var} M_{\Lambda},\left(E_{\Lambda}-\mathbb{E} E_{\Lambda}\right) / \operatorname{Var} E_{\Lambda}\right)$ converges weakly to a standard bivariate Gaussian distribution (i.e., the two components are independent and each is a mean 0 variance 1 Gaussian random variable) if the susceptibility

$$
\chi:=\sum_{j \in \mathbb{Z}^{d}} \operatorname{Cov}\left(\sigma_{0}, \sigma_{j}\right)<\infty .
$$

In particular, this implies that such a convergence holds when $d=2$ and $\beta \neq \beta_{c}$ where $\beta_{c}$ is the critical inverse temperature. A similar Gaussian limit was obtained for the total magnetization and total energy on one side of a rectangle when $d=2$ and $\beta=\beta_{c}$ in De Coninck (1987) (see also Abraham, 1978 for the total magnetization only), and for $\left(M_{\Lambda}, E_{\Lambda}\right)$ when $d>4$ and any $\beta \in\left[0, \beta_{c}\right]$ in De Coninck and Newman (1990). When $d=2$ and $\beta=\beta_{c}$, it was proved in Camia et al. (2015) that $\left(M_{\Lambda}-\mathbb{E} M_{\Lambda}\right) / \operatorname{Var} M_{\Lambda}$ converges weakly to a non-Gaussian limit; and for the Ising model on a $(2 N) \times(2 M)$ rectangle with periodic boundary condition in the horizontal direction and free boundary condition in the vertical direction (with the total energy denoted by $E_{M, N}$ ), it was proved in De Coninck (1984) that $\left(E_{M, N}-\mathbb{E} E_{M, N}\right) / \sqrt{2 M 2 N \ln N}$ converges to a Gaussian distribution by first taking $M \rightarrow \infty$ and then $N \rightarrow \infty$. One disadvantage of this iterated limit is that one does not see the effect from the boundary condition. In this paper, for the same Ising model as considered in De Coninck (1984), we prove a central limit theorem (CLT) for $E_{M, N}$ when both $M$ and $N \rightarrow \infty$ simultaneously. More precisely, we consider the Ising model on $\Lambda_{M, N}:=([1,2 N] \times[1,2 M]) \cap \mathbb{Z}^{2}$ with the total energy

$$
E_{M, N}:=-\sum_{j=1}^{2 M} \sum_{k=1}^{2 N} \sigma_{j, k} \sigma_{j, k+1}-\sum_{j=1}^{2 M-1} \sum_{k=1}^{2 N} \sigma_{j, k} \sigma_{j+1, k}
$$

where $k=2 N+1$ is identified with $k=1$. For each $\sigma \in\{-1,+1\}^{\Lambda_{M, N}}$, we have

$$
\mathbb{P}_{M, N}^{\beta}(\sigma):=\frac{1}{Z_{M, N}(\beta)} e^{-\beta E_{M, N}},
$$

where

$$
Z_{M, N}(\beta):=\sum_{\sigma \in\{-1,+1\}^{\Lambda_{M, N}}} e^{-\beta E_{M, N}}
$$

is the partition function.

Our main result is

Theorem 1.1. Consider the Ising model on $\Lambda_{M, N}$ at critical temperature with periodic boundary condition in the horizontal direction and free boundary condition in the vertical direction (i.e., with the Hamiltonian given by (1.5)). Suppose that $M \in(0, \infty)$ is a function of $N$ satisfying

$$
\lim _{N \rightarrow \infty} \frac{N(\ln \ln N)^{2}}{M \ln N}=0 .
$$

Let $\hat{E}_{M, N}$ be the normalized random variable

$$
\hat{E}_{M, N}:=\frac{E_{M, N}+4 \sqrt{2} M N-(4 / \pi) N \ln N}{\sqrt{4 M N \ln N}} .
$$


Then for each $t \geq 0$,

$$
\lim _{N \rightarrow \infty}\left\langle e^{t \hat{E}_{M, N}}\right\rangle_{M, N}^{\beta_{c}}=e^{4 t^{2} / \pi}
$$

where $\langle\cdot\rangle_{M, N}^{\beta_{c}}$ denotes the expectation with respect to $\mathbb{P}_{M, N}^{\beta_{c}}$. In particular, this implies that $\hat{E}_{M, N}$ converges weakly to a Gaussian distribution with mean 0 and variance $8 / \pi$ as $N \rightarrow \infty$.

Remark 1.2. We believe a similar CLT holds for the critical Ising model with other boundary conditions (e.g., free, all + , all - ). Furthermore, for the critical Ising model on the rescaled lattice $a \mathbb{Z}^{2}$, we expect that the renormalized energy field

$$
a(-\ln a)^{-1 / 2} \sum_{\{x, y\}}\left[\sigma_{x} \sigma_{y}-\sqrt{2} / 2\right] \delta_{(x+y) / 2} \Longrightarrow \text { Gaussian white noise as } a \downarrow 0,
$$

where the sum is over all nearest neighbor pairs in $a \mathbb{Z}^{2}$ and $\delta_{(x+y) / 2}$ is a unit Dirac point measure at $(x+y) / 2$.

Remark 1.3. Let $\Lambda_{a}:=a \mathbb{Z}^{2} \cap \Lambda$ be the $a$-approximation of $\Lambda$. For any $z \in \Lambda$, let $x_{a}(z) y_{a}(z)$ be the edge which is closest to $z$. It was proved in Hongler and Smirnov (2013) that under free or all + boundary condition,

$$
a^{-1}\left[\left\langle\sigma_{x_{a}(z)} \sigma_{y_{a}(z)}\right\rangle_{\Lambda_{a}}^{\beta_{c}}-\sqrt{2} / 2\right]
$$

has a conformally covariant limit as $a \downarrow 0$. See also Hongler (2010) for a generalization of this result to $n$-point energy correlation functions. Even though the results of Hongler and Smirnov (2013); Hongler (2010) do not apply directly to the boundary condition considered in Theorem 1.1, they suggest the $N \ln N$ behavior (resulted from the free boundary condition) in the expectation of $E_{M, N}$ since the limit of (1.12) has an order of $[\operatorname{dist}(z, \partial \Lambda)]^{-1}$ where $\operatorname{dist}(z, \partial \Lambda)$ denotes the Euclidean distance between $z$ and the boundary of $D$.

Remark 1.4. For the full-plane critical Ising model, Hecht (1967) showed that the truncated two-point energy correlation function has the following behavior

$$
\left\langle\epsilon_{z_{1}} \epsilon_{z_{2}}\right\rangle \approx \frac{C}{\left|z_{1}-z_{2}\right|^{2}},
$$

where $\epsilon_{z_{i}}:=\left\langle\sigma_{x\left(z_{i}\right)} \sigma_{y\left(z_{i}\right)}-\sqrt{2} / 2\right\rangle$ with $\left\{x\left(z_{i}\right), y\left(z_{i}\right)\right\}$ the closest edge to $z_{i}$. We remark that a rough computation using (1.13) gives

$$
\operatorname{Var}\left(\hat{E}_{M, N}\right) \approx M N \ln N
$$

which explains the normalization in (1.9). In Di Francesco et al. (1987), by using the continuum Ising conformal field theory, it was shown that (see (2.3), (2.12) and (2.13) there)

$$
\left\langle\epsilon_{z_{1}} \epsilon_{z_{2}} \ldots \epsilon_{z_{2 n}}\right\rangle=\left|\frac{1}{n ! 2^{n}} \sum_{\tau \in S^{2 n}} \prod_{j=1}^{n} A_{\tau(2 j-1) \tau(2 j)}\right|,
$$

where $S^{2 n}$ denotes the set of $(2 n)$ ! permutations of $\{1,2, \ldots, 2 n\}$ and $A_{i j}:=1 /\left(z_{i}-\right.$ $\left.z_{j}\right)^{2}$. Equation (1.15) without the modulus is Isserlis's formula (or Wick's formula) for the multivariate Gaussian distribution. This is one of the motivations of the current paper: the critical scaling limit of the magnetization field was established in Camia et al. (2015) and it is natural to ask if an analogous result holds for the energy field. Theorem 1.1 suggests that a scaling limit of the energy field (with correlations behaving like (1.13) and (1.15)) may not exist in the usual probabilistic 
sense (i.e., pairing the limiting field against some nice test functions to get random variables).

We prove Theorem 1.1 in the next section, our method is similar to that of Abraham (1978); De Coninck (1984, 1987). Namely, we first write the moment generating function of $E_{M, N}$ as a ratio of two partition functions (at different temperatures), and then use the explicit formula for the partition function to derive the asymptotic behavior of this moment generating function.

\section{Proof of the main theorem}

2.1. The partition function. The following lemma about the partition function from McCoy and Wu (1973) is essential to the proof of Theorem 1.1.

Lemma 2.1. The partition function defined in (1.7) is

$$
\begin{aligned}
& Z_{M, N}(\beta) \\
& =(2 \sinh (2 \beta))^{2 M N}(\cosh (\beta))^{-2 N} \prod_{\theta}\left[\frac{e^{2 M \gamma_{\theta}}+e^{-2 M \gamma_{\theta}}}{2}+\frac{e^{2 M \gamma_{\theta}}-e^{-2 M \gamma_{\theta}}}{2} g_{\theta}\right],
\end{aligned}
$$

where the product is over $\theta=\pi(2 n-1) /(2 N)$ with $n=1,2, \ldots, N$, and

$$
\begin{gathered}
\cosh \left(\gamma_{\theta}\right)=\operatorname{coth}(2 \beta) \cosh (2 \beta)-\cos (\theta) \text { with } \gamma_{\theta} \geq 0, \\
g_{\theta}=\frac{\operatorname{coth}(2 \beta)-\cosh (2 \beta) \cos (\theta)}{\sinh \left(\gamma_{\theta}\right)} .
\end{gathered}
$$

Proof: From (3.26) in Chapter VI of McCoy and Wu (1973), we have

$$
\begin{aligned}
Z^{2}= & \left(2 \cosh \beta E_{1}\right)^{8 M N}\left(\cosh \beta E_{2}\right)^{4 N(2 M-1)}(\cosh \beta \mathfrak{H})^{4 N} \\
& \times \prod_{\theta}\left\{\left|1+z_{1} e^{i \theta}\right|^{4 M} \lambda^{2 M}\left[v^{2}\left(1-\frac{i z^{2} v^{\prime}}{z_{2} c v}\right)+\alpha^{-4 M} v^{\prime 2}\left(1+\frac{i z^{2} v}{z_{2} c v^{\prime}}\right)\right]\right\},
\end{aligned}
$$

where the product is over $\theta=\pi(2 n-1) /(2 N)$ with $n=1,2, \ldots, 2 N$. Comparing the Hamiltonian in McCoy and Wu (1973) (see (VI.2.1) of McCoy and Wu (1973)) and our Hamiltonian (1.5), we have

$$
\begin{aligned}
& E_{1}=E_{2}=1, \mathfrak{H}=0, z_{1}=z_{2}=\tanh \beta, z=0, \alpha=e^{\gamma_{\theta}} \\
& \lambda=\frac{\tanh (\beta) \alpha}{\cosh (2 \beta)+\sinh (2 \beta) \cos (\theta)}, v^{2}=\frac{1+g_{\theta}}{2}, v^{\prime 2}=\frac{1-g_{\theta}}{2},
\end{aligned}
$$

where $\gamma_{\theta}$ and $g_{\theta}$ are defined in (2.2) and (2.3) respectively. Plugging (2.5) and (2.6) into (2.4), we get

$$
\left.Z^{2}=(2 \sinh (2 \beta))^{4 M N}\right)(\cosh (\beta))^{-4 N} \prod_{\theta}\left[\frac{e^{2 M \gamma_{\theta}}+e^{-2 M \gamma_{\theta}}}{2}+\frac{e^{2 M \gamma_{\theta}}-e^{-2 M \gamma_{\theta}}}{2} g_{\theta}\right],
$$

where the product is over $\theta=\pi(2 n-1) /(2 N)$ with $n=1,2, \ldots, 2 N$. Equation (2.1) follows from (2.7) and the symmetry of $\theta$.

Remark 2.2. The partition function (2.1) differs from (7) in De Coninck (1984) by a factor of $2^{2 N}$. By checking the particular case $\beta \downarrow 0$, one can see that (2.1) is the correct one. But such a difference does not affect the computation of $\left\langle e^{t E_{M, N}}\right\rangle_{\Lambda_{M, N}}$ since the latter is the ratio of two partition functions (see Lemma 2.4). 
2.2. Proof of Theorem 1.1 modulo Proposition 2.5. It is well-known that the critical inverse temperature for the two-dimensional Ising model is $\beta_{c}=\ln (1+\sqrt{2}) / 2$. We will use the following computations many times in the paper.

Lemma 2.3.

$$
\begin{aligned}
& \sinh \left(2 \beta_{c}\right)=1, \cosh \left(2 \beta_{c}\right)=\sqrt{2},\left.\cosh \left(\gamma_{\theta}\right)\right|_{\beta=\beta_{c}}=2-\cos \theta, \\
& \left.\sinh \left(\gamma_{\theta}\right)\right|_{\beta=\beta_{c}}=\sqrt{3-4 \cos \theta+\cos ^{2} \theta} .
\end{aligned}
$$

Proof: The lemma follows from trivial computations.

Lemma 2.4. For any $s \in \mathbb{R}$ and $\beta \geq 0$,

$$
\left\langle e^{s E_{M, N}}\right\rangle_{M, N}^{\beta}=\frac{Z_{M, N}(\beta-s)}{Z_{M, N}(\beta)} .
$$

Proof:

$$
\left\langle e^{s E_{M, N}}\right\rangle_{M, N}^{\beta}=\frac{\sum_{\sigma} e^{s E_{M, N}} e^{-\beta E_{M, N}}}{Z_{M, N}(\beta)}=\frac{Z_{M, N}(\beta-s)}{Z_{M, N}(\beta)}
$$

By Lemma 2.1, we have

$$
\ln Z_{M, N}(\beta)=2 M N \ln (2 \sinh (2 \beta))-2 N \ln (\cosh (\beta))+2 M \sum_{\theta} \gamma_{\theta}+\sum_{\theta} f_{\theta},
$$

where

$$
f_{\theta}:=\ln \left[1+e^{-4 M \gamma_{\theta}}+\left(1-e^{-4 M \gamma_{\theta}}\right) g_{\theta}\right]-\ln 2 .
$$

We define $L_{i}$ for $i=1,2,3,4$ by

$$
\begin{aligned}
& L_{1}(\beta):=2 M N \ln (2 \sinh (2 \beta)), L_{2}(\beta):=2 N \ln (\cosh (\beta)), \\
& L_{3}(\beta):=2 M \sum_{\theta} \gamma_{\theta}, L_{4}(\beta):=\sum_{\theta} f_{\theta} .
\end{aligned}
$$

In the rest of this paper, we always assume $M$ is a function of $N$ satisfying (1.8). Theorem 1.1 will follow from the following estimates about $L_{i}$ 's.

Proposition 2.5. Suppose $M$ is a function of $N$ satisfying (1.8). Then for each $t \geq 0$, we have

$$
\begin{aligned}
& \lim _{N \rightarrow \infty}\left[L_{1}\left(\beta_{c}-t / \sqrt{4 M N \ln N}\right)-L_{1}\left(\beta_{c}\right)+\frac{t}{\sqrt{4 M N \ln N}} 4 \sqrt{2} M N\right]=0, \\
& \lim _{N \rightarrow \infty}\left[L_{2}\left(\beta_{c}-t / \sqrt{4 M N \ln N}\right)-L_{2}\left(\beta_{c}\right)\right]=0, \\
& \lim _{N \rightarrow \infty}\left[L_{3}\left(\beta_{c}-t / \sqrt{4 M N \ln N}\right)-L_{3}\left(\beta_{c}\right)\right]=\frac{4 t^{2}}{\pi}, \\
& \lim _{N \rightarrow \infty}\left[L_{4}\left(\beta_{c}-t / \sqrt{4 M N \ln N}\right)-L_{4}\left(\beta_{c}\right)-\frac{t}{\sqrt{4 M N \ln N}} \frac{4}{\pi} N \ln N\right]=0 .
\end{aligned}
$$

Let us prove Theorem 1.1 under the assumption of Proposition 2.5.

Proof of Theorem 1.1 (modulo proving Proposition 2.5): For $\hat{E}_{M, N}$ defined in (1.9), we have by Lemma 2.4 that

$$
\ln \left\langle e^{t \hat{E}_{M, N}}\right\rangle_{M, N}^{\beta_{c}}
$$




$$
\begin{aligned}
= & \ln \left\langle e^{t E_{M, N} / \sqrt{4 M N \ln N}}\right\rangle_{M, N}^{\beta_{c}}+\frac{t}{\sqrt{4 M N \ln N}}\left[4 \sqrt{2} M N-\frac{4}{\pi} N \ln N\right] \\
= & \ln Z_{M, N}\left(\beta_{c}-t / \sqrt{4 M N \ln N}\right)-\ln Z_{M, N}\left(\beta_{c}\right) \\
& +\frac{t}{\sqrt{4 M N \ln N}}\left[4 \sqrt{2} M N-\frac{4}{\pi} N \ln N\right] .
\end{aligned}
$$

Using (2.12)-(2.15), we can write

$$
\begin{aligned}
& \ln \left\langle e^{t \hat{E}_{M, N}}\right\rangle_{M, N}^{\beta_{c}}=\left[L_{1}\left(\beta_{c}-t / \sqrt{4 M N \ln N}\right)-L_{1}\left(\beta_{c}\right)+\frac{t}{\sqrt{4 M N \ln N}} 4 \sqrt{2} M N\right] \\
& -\left[L_{2}\left(\beta_{c}-t / \sqrt{4 M N \ln N}\right)-L_{2}\left(\beta_{c}\right)\right]+\left[L_{3}\left(\beta_{c}-t / \sqrt{4 M N \ln N}\right)-L_{3}\left(\beta_{c}\right)\right] \\
& +\left[L_{4}\left(\beta_{c}-t / \sqrt{4 M N \ln N}\right)-L_{4}\left(\beta_{c}\right)-\frac{t}{\sqrt{4 M N \ln N}} \frac{4}{\pi} N \ln N\right] .
\end{aligned}
$$

This completes the proof of the first part of Theorem 1.1 (i.e., (1.10)) by applying Proposition 2.5. The second part of Theorem 1.1 follows from a standard probability argument (see, e.g., Problem 30.4 of Billingsley, 1995).

2.3. Proof of Proposition 2.5. The first two limits in Proposition 2.5 are easy to prove.

Proof of (2.16) and (2.17) in Proposition 2.5: Note that $L_{1}^{\prime}(\beta)=4 M N \operatorname{coth}(2 \beta)$ and $L_{1}^{\prime \prime}(\beta)=-8 M N \operatorname{csch}^{2}(2 \beta)$. So by the Taylor expansion of $L_{1}$ around $\beta_{c}$ and Lemma 2.3 , we have

$$
L_{1}\left(\beta_{c}-t / \sqrt{4 M N \ln N}\right)-L_{1}\left(\beta_{c}\right)=-\frac{t}{\sqrt{4 M N \ln N}} 4 \sqrt{2} M N+\frac{t^{2}}{8 M N \ln N} L_{1}^{\prime \prime}(\tilde{\beta}),
$$

where $\tilde{\beta} \in\left(\beta_{c}-t / \sqrt{4 M N \ln N}, \beta_{c}\right)$. By Lemma $2.3,\left|L_{1}^{\prime \prime}(\tilde{\beta})\right|=8 M N \operatorname{csch}^{2}(2 \tilde{\beta}) \leq$ $16 M N$ for any $\tilde{\beta} \in\left(\beta_{c}-t / \sqrt{4 M N \ln N}, \beta_{c}\right)$ if $N$ is large. This completes the proof of (2.16). Similarly, the Taylor expansion of $L_{2}$ around $\beta_{c}$ gives

$$
L_{2}\left(\beta_{c}-t / \sqrt{4 M N \ln N}\right)-L_{2}\left(\beta_{c}\right)=-\frac{t}{\sqrt{4 M N \ln N}} 2 N \tanh (\tilde{\beta})
$$

where $\tilde{\beta} \in\left(\beta_{c}-t / \sqrt{4 M N \ln N}, \beta_{c}\right)$. It is clear that $|\tanh (\tilde{\beta})| \leq 1$ for any such $\tilde{\beta}$ whenever $N$ is large. Combining this and our assumption on $M$ (i.e., (1.8)) completes the proof of (2.17).

The following three lemmas will be very useful when we deal with the Taylor expansions of $L_{3}(\beta)$ and $L_{4}(\beta)$.

\section{Lemma 2.6.}

$$
\inf _{\beta>0} \operatorname{coth}(2 \beta) \cosh (2 \beta)=2 \text { with the infimum achieved at } \beta=\beta_{c} \text {. }
$$

For each large $N$, each $\beta \in\left(\beta_{c}-1 / \sqrt{4 M N \ln N}, \beta_{c}+1 / \sqrt{4 M N \ln N}\right)$ and each $\theta \in(0, \pi]$, we have

$$
|1-\operatorname{csch}(2 \beta)| \leq 8 / \sqrt{4 M N \ln N}
$$




$$
\begin{array}{r}
\left|[1-\operatorname{csch}(2 \beta)] \operatorname{csch}\left(\gamma_{\theta}\right)\right| \leq \sqrt{2}, \\
\left|[\operatorname{csch}(2 \beta)-\cos \theta] \operatorname{csch}\left(\gamma_{\theta}\right)\right| \leq 3 .
\end{array}
$$

Proof: The proof of (2.22) is trivial. The inequality (2.23) follows from Lemma 2.3 and the mean value theorem. The inequality (2.24) follows from

$$
\begin{aligned}
& \left|[1-\operatorname{csch}(2 \beta)] \operatorname{csch}\left(\gamma_{\theta}\right)\right| \\
= & \frac{|1-\operatorname{csch}(2 \beta)|}{\sqrt{[\operatorname{coth}(2 \beta) \cosh (2 \beta)+1-\cos \theta][\operatorname{coth}(2 \beta) \cosh (2 \beta)-1-\cos \theta]}} \\
\leq & \frac{1}{\sqrt{2}} \frac{|1-\operatorname{csch}(2 \beta)|}{\sqrt{\operatorname{coth}(2 \beta) \cosh (2 \beta)-2}} \quad \text { by }(2.22)
\end{aligned}
$$

and

$$
\lim _{\beta \rightarrow \beta_{c}} \frac{|1-\operatorname{csch}(2 \beta)|}{\sqrt{\operatorname{coth}(2 \beta) \cosh (2 \beta)-2}}=1 .
$$

The inequality (2.25) follows from

$$
\begin{aligned}
& \left|[\operatorname{csch}(2 \beta)-\cos \theta] \operatorname{csch}\left(\gamma_{\theta}\right)\right| \\
= & \frac{|\operatorname{csch}(2 \beta)-\cos \theta|}{\sqrt{[\operatorname{coth}(2 \beta) \cosh (2 \beta)+1-\cos \theta][\operatorname{coth}(2 \beta) \cosh (2 \beta)-1-\cos \theta]}} \\
\leq & \frac{1}{\sqrt{2}} \frac{|\operatorname{csch}(2 \beta)-1|+|1-\cos \theta|}{\sqrt{\operatorname{coth}(2 \beta) \cosh (2 \beta)-2+1-\cos \theta}} \quad \text { by }(2.22) \\
\leq & \frac{1}{\sqrt{2}} \frac{|\operatorname{csch}(2 \beta)-1|+|1-\cos \theta|}{\sqrt{\max \{\operatorname{coth}(2 \beta) \cosh (2 \beta)-2,1-\cos \theta\}}} \\
\leq & \frac{1}{\sqrt{2}}\left[\frac{|\operatorname{csch}(2 \beta)-1|}{\sqrt{\operatorname{coth}(2 \beta) \cosh (2 \beta)-2}}+\frac{|1-\cos \theta|}{\sqrt{1-\cos \theta}}\right]
\end{aligned}
$$

and (2.26).

Lemma 2.7. There exist constants $C_{1}, C_{2} \in(0, \infty)$ such that for all large $N \in \mathbb{N}$,

$$
\left|\sum_{n=1}^{N} \frac{1}{2 n-1}-\frac{\ln N}{2}\right| \leq C_{1},\left|\sum_{n=\lfloor\ln N\rfloor+1}^{N} \frac{1}{2 n-1}-\frac{\ln N}{2}\right| \leq C_{2} \ln \ln N .
$$

Proof: Let $H_{n}:=\sum_{k=1}^{n} 1 / k$ be the $n$-th harmonic number. It is well-known that

$$
\frac{1}{2(n+1)} \leq H_{n}-\ln n-\hat{\gamma} \leq \frac{1}{2 n} \text { for each } n \in \mathbb{N},
$$

where $\hat{\gamma}$ is the Euler-Mascheroni constant. The lemma follows by the following observation:

$$
\begin{aligned}
\sum_{n=1}^{N} \frac{1}{2 n-1}=H_{2 N}-\frac{H_{N}}{2} \\
\quad \sum_{n=\lfloor\ln N\rfloor+1}^{N} \frac{1}{2 n-1}=H_{2 N}-\frac{H_{N}}{2}-\left[H_{2\lfloor\ln N\rfloor}-\frac{H_{\lfloor\ln N\rfloor}}{2}\right] .
\end{aligned}
$$


Lemma 2.8. There exist constants $C_{3}, C_{4} \in(0, \infty)$ such that for all large $N$ and all $\beta>0$,

$$
\sum_{\theta} \operatorname{csch}\left(\gamma_{\theta}\right) \leq C_{3} N \ln N, \quad \sum_{\theta} \operatorname{csch}^{2}\left(\gamma_{\theta}\right) \leq C_{4} N^{2} .
$$

Proof: Recall that

$$
\operatorname{csch}\left(\gamma_{\theta}\right)=\frac{1}{\sqrt{[\operatorname{coth}(2 \beta) \cosh (2 \beta)+1-\cos \theta][\operatorname{coth}(2 \beta) \cosh (2 \beta)-1-\cos \theta]}} .
$$

By (2.22) in Lemma 2.6,

$$
\operatorname{csch}\left(\gamma_{\theta}\right) \leq \frac{1}{\sqrt{(3-\cos \theta)(1-\cos \theta)}} \leq \frac{1}{\sqrt{2(1-\cos \theta)}} \leq \frac{2}{\theta},
$$

where the last inequality follows since

$$
1-\cos x \geq x^{2} / 8 \text { for each } x \in[0, \pi] .
$$

Therefore, by Lemma 2.7 and $\sum_{n=1}^{\infty} 1 /(2 n-1)^{2}<\infty$, for all large $N$,

$$
\begin{gathered}
\sum_{\theta} \operatorname{csch}\left(\gamma_{\theta}\right) \leq \sum_{\theta} \frac{2}{\theta}=\frac{4 N}{\pi} \sum_{n=1}^{N} \frac{1}{2 n-1} \leq C_{3} N \ln N, \\
\sum_{\theta} \operatorname{csch}^{2}\left(\gamma_{\theta}\right) \leq \sum_{\theta} \frac{4}{\theta^{2}}=\frac{16 N^{2}}{\pi^{2}} \sum_{n=1}^{N} \frac{1}{(2 n-1)^{2}} \leq C_{4} N^{2} .
\end{gathered}
$$

From (2.2), we can compute (all derivatives are respect to $\beta$ and here the grouping and ordering of terms is chosen in view of Lemma 2.6)

$$
\begin{gathered}
\gamma_{\theta}^{\prime}=2 \cosh (2 \beta)\left[1-\operatorname{csch}^{2}(2 \beta)\right] \operatorname{csch}\left(\gamma_{\theta}\right), \\
\gamma_{\theta}^{\prime \prime}=4 \sinh (2 \beta)\left[1-\operatorname{csch}^{2}(2 \beta)\right] \operatorname{csch}\left(\gamma_{\theta}\right)+8 \cosh ^{2}(2 \beta) \operatorname{csch}^{3}(2 \beta) \operatorname{csch}\left(\gamma_{\theta}\right) \\
-4 \cosh ^{2}(2 \beta)\left[1-\operatorname{csch}^{2}(2 \beta)\right]^{2} \cosh \left(\gamma_{\theta}\right) \operatorname{csch}^{3}\left(\gamma_{\theta}\right), \\
\gamma_{\theta}^{\prime \prime \prime}=\left\{8 \cosh (2 \beta)\left[1-\operatorname{csch}^{2}(2 \beta)\right] \operatorname{csch}\left(\gamma_{\theta}\right)-8 \cosh ^{3}(2 \beta)\left[1-\operatorname{csch}^{2}(2 \beta)\right]^{3} \operatorname{csch}^{3}\left(\gamma_{\theta}\right)\right\} \\
+\left\{16 \operatorname{csch}(2 \beta) \operatorname{coth}(2 \beta)-24 \cosh (2 \beta) \sinh (2 \beta) \cosh \left(\gamma_{\theta}\right)\left[1-\operatorname{csch}^{2}(2 \beta)\right]^{2} \operatorname{csch}^{2}\left(\gamma_{\theta}\right)\right. \\
\left.+32 \cosh (2 \beta) \operatorname{csch}^{2}(2 \beta)-48 \cosh ^{3}(2 \beta) \operatorname{csch}^{4}(2 \beta)\right\} \operatorname{csch}\left(\gamma_{\theta}\right) \\
+\left\{-48 \cosh (2 \beta) \operatorname{csch}^{3}(2 \beta) \cosh \left(\gamma_{\theta}\right)\left[1-\operatorname{csch}^{2}(2 \beta)\right] \operatorname{csch}\left(\gamma_{\theta}\right)\right. \\
\left.+24 \cosh ^{3}(2 \beta) \cosh ^{2}\left(\gamma_{\theta}\right)\left[1-\operatorname{csch}^{2}(2 \beta)\right]^{3} \operatorname{csch}^{3}\left(\gamma_{\theta}\right)\right\} \operatorname{csch}^{2}\left(\gamma_{\theta}\right) .
\end{gathered}
$$

By Lemma 2.3, we have

$$
\left.\gamma_{\theta}^{\prime}\right|_{\beta=\beta_{c}}=0,\left.\gamma_{\theta}^{\prime \prime}\right|_{\beta=\beta_{c}}=\frac{16}{\sqrt{3-4 \cos \theta+\cos ^{2} \theta}} .
$$

We are ready to prove (2.18) in Proposition 2.5. 
Proof of (2.18) in Proposition 2.5: The Taylor expansion of $L_{3}$ (see (2.15)) around $\beta=\beta_{c}$ implies that there exists $\tilde{\beta} \in\left(\beta_{c}-t / \sqrt{4 M N \ln N}, \beta_{c}\right)$ such that

$$
\begin{aligned}
& L_{3}\left(\beta_{c}-t / \sqrt{4 M N \ln N}\right)-L_{3}\left(\beta_{c}\right) \\
= & \frac{-t}{\sqrt{4 M N \ln N}} L_{3}^{\prime}\left(\beta_{c}\right)+\frac{t^{2}}{8 M N \ln N} L_{3}^{\prime \prime}\left(\beta_{c}\right)-\frac{t^{3}}{6(4 M N \ln N)^{3 / 2}} L_{3}^{\prime \prime \prime}(\tilde{\beta}) \\
= & \frac{t^{2}}{4 N \ln N} \sum_{\theta} \frac{16}{\sqrt{3-4 \cos \theta+\cos ^{2} \theta}}-\left.\frac{t^{3} M}{3(4 M N \ln N)^{3 / 2}} \sum_{\theta} \gamma_{\theta}^{\prime \prime \prime}\right|_{\beta=\tilde{\beta}},
\end{aligned}
$$

where we have used (2.35) in the last equality.

Note that $1 / \sqrt{3-4 \cos \theta+\cos ^{2} \theta}-1 / \theta$ is a continuous function of $\theta$ on $[0, \pi]$ if we define its value at $\theta=0$ being 0 since

$$
\lim _{\theta \downarrow 0}\left[\frac{1}{\sqrt{3-4 \cos \theta+\cos ^{2} \theta}}-\frac{1}{\theta}\right]=0 .
$$

Therefore, there exists a constant $C_{5} \in(0, \infty)$ such that for all $N \in \mathbb{N}$,

$$
\sum_{\theta}\left|\frac{1}{\sqrt{3-4 \cos \theta+\cos ^{2} \theta}}-\frac{1}{\theta}\right| \leq C_{5} N
$$

where we have used that each summand is bounded by $C_{5}$. Now we have

$$
\begin{aligned}
& \lim _{N \rightarrow \infty} \frac{t^{2}}{4 N \ln N} \sum_{\theta} \frac{16}{\sqrt{3-4 \cos \theta+\cos ^{2} \theta}} \\
= & \lim _{N \rightarrow \infty} \frac{4 t^{2}}{N \ln N}\left\{\sum_{\theta}\left[\frac{1}{\sqrt{3-4 \cos \theta+\cos ^{2} \theta}}-\frac{1}{\theta}\right]+\sum_{\theta} \frac{1}{\theta}\right\} \\
= & \lim _{N \rightarrow \infty} \frac{4 t^{2}}{N \ln N} \frac{2 N}{\pi} \sum_{n=1}^{N} \frac{1}{2 n-1} \\
= & \frac{4 t^{2}}{\pi},
\end{aligned}
$$

where we have used $(2.37)$ and $\theta=(2 n-1) \pi /(2 N)$ in the second equality and Lemma 2.7 in the last equality.

Next, we prove that the remainder in (2.36) vanishes as $N \rightarrow \infty$. By (2.34), Lemmas 2.3 and 2.6, there exist constants $C_{6}, C_{7}, C_{8} \in(0, \infty)$ such that for all large $N$, each $\tilde{\beta} \in\left(\beta_{c}-t / \sqrt{4 M N \ln N}, \beta_{c}\right)$ and each $\theta \in(0, \pi]$,

$$
\left|\gamma_{\theta}^{\prime \prime \prime}\right|_{\beta=\tilde{\beta}}\left|\leq C_{6}+C_{7} \operatorname{csch}\left(\gamma_{\theta}\right)\right|_{\beta=\tilde{\beta}}+\left.C_{8} \operatorname{csch}^{2}\left(\gamma_{\theta}\right)\right|_{\beta=\tilde{\beta}}
$$

Therefore, by Lemma 2.8, we have for all large $N$,

$$
\left.\left|\sum_{\theta} \gamma_{\theta}^{\prime \prime \prime}\right|_{\beta=\tilde{\beta}}\left|\leq \sum_{\theta}\right| \gamma_{\theta}^{\prime \prime \prime}\right|_{\beta=\tilde{\beta}} \mid \leq C_{6} N+C_{7} C_{3} N \ln N+C_{8} C_{4} N^{2} .
$$


This and (1.8) imply

$$
\begin{aligned}
& \lim _{N \rightarrow \infty} \frac{t^{3} M}{3(4 M N \ln N)^{3 / 2}}\left|\sum_{\theta} \gamma_{\theta}^{\prime \prime \prime}\right|_{\beta=\tilde{\beta}} \mid \\
& \leq \lim _{N \rightarrow \infty} \frac{t^{3} M\left[C_{6} N+C_{7} C_{3} N \ln N+C_{8} C_{4} N^{2}\right]}{3(4 M N \ln N)^{3 / 2}}=0 .
\end{aligned}
$$

Combining (2.36), (2.38) and (2.39), we finish the proof of (2.18).

The last and more difficult function we need to deal with is $L_{4}(\beta)=\sum_{\theta} f_{\theta}$. We first compute the derivatives of $f_{\theta}$ (with respect to $\beta$ ). By (2.13), (2.2), (2.3) and (2.32), we have

$$
\begin{aligned}
f_{\theta}^{\prime}= & \frac{4 M \gamma_{\theta}^{\prime} e^{-4 M \gamma_{\theta}}\left(g_{\theta}-1\right)+\left(1-e^{-4 M \gamma_{\theta}}\right) g_{\theta}^{\prime}}{1+e^{-4 M \gamma_{\theta}}+\left(1-e^{-4 M \gamma_{\theta}}\right) g_{\theta}} \\
f_{\theta}^{\prime \prime}= & \frac{4 M \gamma_{\theta}^{\prime \prime} e^{-4 M \gamma_{\theta}}\left(g_{\theta}-1\right)-16 M^{2}\left(\gamma_{\theta}^{\prime}\right)^{2} e^{-4 M \gamma_{\theta}}\left(g_{\theta}-1\right)+8 M \gamma_{\theta}^{\prime} e^{-4 M \gamma_{\theta}} g_{\theta}^{\prime}+\left(1-e^{-4 M \gamma_{\theta}}\right) g_{\theta}^{\prime \prime}}{1+e^{-4 M \gamma_{\theta}}+\left(1-e^{-4 M \gamma_{\theta}}\right) g_{\theta}} \\
& -\left[\frac{4 M \gamma_{\theta}^{\prime} e^{-4 M \gamma_{\theta}}\left(g_{\theta}-1\right)+\left(1-e^{-4 M \gamma_{\theta}}\right) g_{\theta}^{\prime}}{1+e^{-4 M \gamma_{\theta}}+\left(1-e^{-4 M \gamma_{\theta}}\right) g_{\theta}}\right]^{2}
\end{aligned}
$$

where (here again the grouping and ordering of terms is chosen in view of Lemma 2.6)

$$
\begin{aligned}
& g_{\theta}^{\prime}=-2\left[\operatorname{csch}^{2}(2 \beta)+\sinh (2 \beta) \cos \theta\right] \operatorname{csch}\left(\gamma_{\theta}\right) \\
&- 2 \cosh ^{2}(2 \beta)[\operatorname{coth}(2 \beta) \cosh (2 \beta)-\cos \theta]\left[1-\operatorname{csch}^{2}(2 \beta)\right][\operatorname{csch}(2 \beta)-\cos \theta] \operatorname{csch}^{3}\left(\gamma_{\theta}\right) \\
& g_{\theta}^{\prime \prime}=\left\{-4 \cosh ^{3}(2 \beta)\left[1-\operatorname{csch}^{2}(2 \beta)\right]^{2}[\operatorname{csch}(2 \beta)-\cos \theta] \operatorname{csch}^{3}\left(\gamma_{\theta}\right)\right\} \\
&+\left\{8 \operatorname{csch}^{2}(2 \beta) \operatorname{coth}(2 \beta)-4 \cosh (2 \beta) \cos \theta-8 \cosh (2 \beta) \sinh (2 \beta) \times\right. \\
&\left.\quad[\operatorname{coth}(2 \beta) \cosh (2 \beta)-\cos \theta]\left[1-\operatorname{csch}^{2}(2 \beta)\right][\operatorname{csch}(2 \beta)-\cos \theta] \operatorname{csch}^{2}\left(\gamma_{\theta}\right)\right\} \operatorname{csch}\left(\gamma_{\theta}\right) \\
&+\left\{4 \cosh (2 \beta)[\operatorname{csch}(2 \beta)+\sinh (2 \beta) \cos \theta] \cosh \left(\gamma_{\theta}\right)[1-\operatorname{csch}(2 \beta)] \operatorname{csch}\left(\gamma_{\theta}\right)\right. \\
& \quad-8 \cosh (2 \beta) \operatorname{csch}^{3}(2 \beta)[\operatorname{coth}(2 \beta) \cosh (2 \beta)-\cos \theta]\left[\operatorname{csch}^{2}(2 \beta)-\cos _{\theta} \theta \operatorname{csch}\left(\gamma_{\theta}\right)\right. \\
& \quad+4 \cosh ^{3}(2 \beta) \operatorname{csch}^{2}(2 \beta)[\operatorname{coth}(2 \beta) \cosh (2 \beta)-\cos \theta]\left[1-\operatorname{csch}^{2}(2 \beta)\right] \operatorname{csch}\left(\gamma_{\theta}\right) \\
&+12 \cosh ^{3}(2 \beta)\left[\operatorname{coth}^{2}(2 \beta) \cosh (2 \beta)-\cos \theta\right] \cosh \left(\gamma_{\theta}\right)\left[1-\operatorname{csch}^{2}(2 \beta)\right]^{2} \times \\
&\left.\quad\left[\operatorname{csch}^{2}(2 \beta)-\cos \theta\right] \operatorname{csch}^{3}\left(\gamma_{\theta}\right)\right\} \operatorname{csch}^{2}\left(\gamma_{\theta}\right) .
\end{aligned}
$$

By Lemma 2.3, (2.3), (2.35), (2.40) and (2.42), we have

$$
\left.f_{\theta}^{\prime}\right|_{\beta=\beta_{c}}=\frac{-2\left(1-\eta_{\theta}^{-4 M}\right)(1+\cos \theta)(3-\cos \theta)^{-1 / 2}(1-\cos \theta)^{-1 / 2}}{1+\eta_{\theta}^{-4 M}+\left(1-\eta_{\theta}^{-4 M}\right) \sqrt{2}(1-\cos \theta)^{1 / 2}(3-\cos \theta)^{-1 / 2}},
$$

where

$$
\eta_{\theta}:=\left.e^{\gamma_{\theta}}\right|_{\beta=\beta_{c}}=\left(\frac{\sqrt{3-\cos \theta}+\sqrt{1-\cos \theta}}{\sqrt{2}}\right)^{2} \geq 1 .
$$

We need the following lemma to analyze the Taylor expansion of $L^{4}(\beta)$ around $\beta_{c}$. Let us emphasize again that $M$ is a function of $N$ satisfying (1.8). 
Lemma 2.9. There exist constants $C_{9}, C_{10} \in(0, \infty)$ such that for each $\beta>0$ and each large $N$,

$$
\begin{aligned}
& \sum_{\theta}\left(e^{-4 M \gamma_{\theta}} \theta^{-1}\right) \leq C_{9} N \ln \ln N, \\
& \sum_{\theta}\left[e^{-4 M \gamma_{\theta}} \operatorname{csch}\left(\gamma_{\theta}\right)\right] \leq C_{10} N \ln \ln N .
\end{aligned}
$$

Proof: By (2.2), and (2.22) in Lemma 2.6, we have

$$
\begin{aligned}
& \sum_{\theta}\left(e^{-4 M \gamma_{\theta}} \theta^{-1}\right) \\
= & \sum_{\theta}\left[\left(\frac{\sqrt{\operatorname{coth}(2 \beta) \cosh (2 \beta)+1-\cos \theta}+\sqrt{\operatorname{coth}(2 \beta) \cosh (2 \beta)-1-\cos \theta}}{\sqrt{2}}\right)^{-8 M} \theta^{-1}\right] \\
\leq & \sum_{\theta}\left[\left(\frac{\sqrt{3-\cos \theta}+\sqrt{1-\cos \theta}}{\sqrt{2}}\right)^{-8 M} \theta^{-1}\right] \\
\leq & \frac{2 N}{\pi} \sum_{n=1}^{\lfloor\ln N\rfloor} \frac{1}{2 n-1} \\
& +\frac{2 N}{\pi} \sum_{n=\lfloor\ln N\rfloor+1}^{N}\left[\left(\frac{\sqrt{3-\cos \left(\frac{(2 n-1) \pi}{2 N}\right)}+\sqrt{1-\cos \left(\frac{(2 n-1) \pi}{2 N}\right)}}{\sqrt{2}}\right)^{-8 M} \frac{1}{2 n-1}\right]
\end{aligned}
$$

where the last inequality follows since $(\sqrt{3-\cos \theta}+\sqrt{1-\cos \theta}) / \sqrt{2} \geq 1$ for any $\theta \in[0, \pi]$. It is easy to see that (see, e.g., the proof of Lemma 2.7)

$$
\frac{2 N}{\pi} \sum_{n=1}^{\lfloor\ln N\rfloor} \frac{1}{2 n-1} \leq N \ln \ln N \text { for all large } N .
$$

For the other sum in the RHS of (2.48), we have (using (2.31) in the second inequality)

$$
\begin{aligned}
& \sum_{n=\lfloor\ln N\rfloor+1}^{N}\left[\left(\frac{\sqrt{3-\cos \left(\frac{(2 n-1) \pi}{2 N}\right)}+\sqrt{1-\cos \left(\frac{(2 n-1) \pi}{2 N}\right)}}{\sqrt{2}}\right)\right. \\
\leq & \sum_{n=\lfloor\ln N\rfloor+1}^{N}\left[\left(\frac{\sqrt{2}+\sqrt{1-\cos (\pi\lfloor\ln N\rfloor / N)}}{\sqrt{2}}\right)^{-8 M} \frac{1}{2 n-1}\right] \\
\leq & \sum_{n=\lfloor\ln N\rfloor+1}^{N}\left[\left(\frac{\sqrt{2}+\sqrt{(\pi\lfloor\ln N\rfloor / N)^{2} / 8}}{\sqrt{2}}\right)^{-8 M} \frac{1}{2 n-1}\right] \\
= & \left(1+\frac{\pi\lfloor\ln N\rfloor}{4 N}\right)^{-8 M} \sum_{n=\lfloor\ln N\rfloor+1}^{N} \frac{1}{2 n-1}
\end{aligned}
$$




$$
\leq e^{-\pi M\lfloor\ln N\rfloor / N} \ln N \text { for all large } N,
$$

where the the last inequality follows from Lemma 2.7 and

$$
\left(1+\frac{\pi\lfloor\ln N\rfloor}{4 N}\right)^{4 N /(\pi\lfloor\ln N\rfloor)} \geq e^{1 / 2} \text { for all large } N .
$$

From (1.8), we have

$$
e^{-\pi M\lfloor\ln N\rfloor / N} \leq e^{-\ln \ln N}=(\ln N)^{-1} \text { for all large } N .
$$

Combining (2.48)-(2.51), we get (2.46). The inequality (2.47) follows from (2.30) and (2.46).

The Taylor expansion of $L_{4}($ see $(2.15))$ around $\beta=\beta_{c}$ gives

$$
\begin{aligned}
& L_{4}\left(\beta_{c}-t / \sqrt{4 M N \ln N}\right)-L_{4}\left(\beta_{c}\right) \\
& =\left.\frac{-t}{\sqrt{4 M N \ln N}} \sum_{\theta} f_{\theta}^{\prime}\right|_{\beta=\beta_{c}}+\left.\frac{t^{2}}{8 M N \ln N} \sum_{\theta} f_{\theta}^{\prime \prime}\right|_{\beta=\tilde{\beta}}
\end{aligned}
$$

where $\tilde{\beta} \in\left(\beta_{c}-t / \sqrt{4 M N \ln N}, \beta_{c}\right)$. The following lemma is about the asymptotic behavior of the first term on the RHS of (2.52).

Lemma 2.10.

$$
\lim _{N \rightarrow \infty} \frac{-t}{\sqrt{4 M N \ln N}}\left[\left.\sum_{\theta} f_{\theta}^{\prime}\right|_{\beta=\beta_{c}}+\frac{4}{\pi} N \ln N\right]=0 .
$$

Proof: From (2.44), we have (recall that $\eta_{\theta} \geq 1$ from $(2.45)$ )

$$
\begin{aligned}
& -\left.\sum_{\theta} f_{\theta}^{\prime}\right|_{\beta=\beta_{c}}=\sum_{\theta} \frac{2\left(1-\eta_{\theta}^{-4 M}\right)(1+\cos \theta)(3-\cos \theta)^{-1 / 2}(1-\cos \theta)^{-1 / 2}}{1+\eta_{\theta}^{-4 M}+\left(1-\eta_{\theta}^{-4 M}\right) \sqrt{2}(1-\cos \theta)^{1 / 2}(3-\cos \theta)^{-1 / 2}} \\
& =\sum_{\theta} \frac{2\left(1-\eta_{\theta}^{-4 M}\right)(1+\cos \theta)(3-\cos \theta)^{-1 / 2}\left[(1-\cos \theta)^{-1 / 2}-\sqrt{2} \theta^{-1}\right]}{1+\eta_{\theta}^{-4 M}+\left(1-\eta_{\theta}^{-4 M}\right) \sqrt{2}(1-\cos \theta)^{1 / 2}(3-\cos \theta)^{-1 / 2}} \\
& +\sum_{\theta} \frac{2\left(1-\eta_{\theta}^{-4 M}\right)(1+\cos \theta)(3-\cos \theta)^{-1 / 2} \sqrt{2} \theta^{-1}}{1+\eta_{\theta}^{-4 M}+\left(1-\eta_{\theta}^{-4 M}\right) \sqrt{2}(1-\cos \theta)^{1 / 2}(3-\cos \theta)^{-1 / 2}} .
\end{aligned}
$$

Since $\lim _{\theta \downarrow 0}\left[(1-\cos \theta)^{-1 / 2}-\sqrt{2} \theta^{-1}\right]=0$, there exists a constant $C_{11} \in(0, \infty)$ such that for each $N \in \mathbb{N}$,

$$
\left|\sum_{\theta} \frac{2\left(1-\eta_{\theta}^{-4 M}\right)(1+\cos \theta)(3-\cos \theta)^{-1 / 2}\left[(1-\cos \theta)^{-1 / 2}-\sqrt{2} \theta^{-1}\right]}{1+\eta_{\theta}^{-4 M}+\left(1-\eta_{\theta}^{-4 M}\right) \sqrt{2}(1-\cos \theta)^{1 / 2}(3-\cos \theta)^{-1 / 2}}\right| \leq C_{11} N
$$

The last sum in the RHS of (2.54) contains

$$
\begin{aligned}
& \sum_{\theta} \frac{2 \eta_{\theta}^{-4 M}(1+\cos \theta)(3-\cos \theta)^{-1 / 2} \sqrt{2} \theta^{-1}}{1+\eta_{\theta}^{-4 M}+\left(1-\eta_{\theta}^{-4 M}\right) \sqrt{2}(1-\cos \theta)^{1 / 2}(3-\cos \theta)^{-1 / 2}} \leq 4 \sum_{\theta}\left[\eta_{\theta}^{-4 M^{-1}} \theta^{-1}\right] \\
= & 4 \sum_{\theta}\left[\left.e^{-4 M \gamma_{\theta}}\right|_{\beta=\beta_{c}} \theta^{-1}\right] \leq 4 C_{9} N \ln \ln N,
\end{aligned}
$$


where we have used (2.45), and (2.46) from Lemma 2.9 in the last equality. The remaining sum that we have not analyzed is

$$
\begin{aligned}
& \sum_{\theta} \frac{2(1+\cos \theta)(3-\cos \theta)^{-1 / 2} \sqrt{2} \theta^{-1}}{1+\eta_{\theta}^{-4 M}+\left(1-\eta_{\theta}^{-4 M}\right) \sqrt{2}(1-\cos \theta)^{1 / 2}(3-\cos \theta)^{-1 / 2}} \\
= & \sum_{\theta} \frac{2(1+\cos \theta)(3-\cos \theta)^{-1 / 2} \sqrt{2} \theta^{-1}\left[-\eta_{\theta}^{-4 M}\left(1-\sqrt{\frac{2(1-\cos \theta)}{(3-\cos \theta)}}\right)\right]}{\left[1+\sqrt{\frac{2(1-\cos \theta)}{(3-\cos \theta)}}\right]\left[1+\sqrt{\frac{2(1-\cos \theta)}{(3-\cos \theta)}}+\eta_{\theta}^{-4 M}\left(1-\sqrt{\frac{2(1-\cos \theta)}{(3-\cos \theta)}}\right)\right]} \\
& +\sum_{\theta} \frac{2(1+\cos \theta)(3-\cos \theta)^{-1 / 2} \sqrt{2} \theta^{-1}}{1+\sqrt{2}(1-\cos \theta)^{1 / 2}(3-\cos \theta)^{-1 / 2}} .
\end{aligned}
$$

By applying (2.46) from Lemma 2.9, we get (noting that $\sqrt{2(1-\cos x) /(3-\cos x)}$ $\in[0,1])$

$$
\begin{aligned}
& \mid \sum_{\theta} \frac{2(1+\cos \theta)(3-\cos \theta)^{-1 / 2} \sqrt{2} \theta^{-1}\left[-\eta_{\theta}^{-4 M}\left(1-\sqrt{\frac{2(1-\cos \theta)}{(3-\cos \theta)}}\right)\right]}{\left[1+\sqrt{\left.\frac{2(1-\cos \theta)}{(3-\cos \theta)}\right]\left[1+\sqrt{\frac{2(1-\cos \theta)}{(3-\cos \theta)}}+\eta_{\theta}^{-4 M}\left(1-\sqrt{\frac{2(1-\cos \theta)}{(3-\cos \theta)}}\right)\right]} \mid\right.} \\
& \leq 4 \sum_{\theta}\left[\eta_{\theta}^{-4 M} \theta^{-1}\right]=\sum_{\theta}\left[\left.e^{-4 M \gamma_{\theta}}\right|_{\beta=\beta_{c}} \theta^{-1}\right] \leq 4 C_{9} N \ln \ln N .
\end{aligned}
$$

The second sum on the RHS of (2.57) is

$$
\begin{aligned}
\sum_{\theta} & \frac{2(1+\cos \theta)(3-\cos \theta)^{-1 / 2} \sqrt{2} \theta^{-1}}{1+\sqrt{2}(1-\cos \theta)^{1 / 2}(3-\cos \theta)^{-1 / 2}}=\sum_{\theta} \frac{4}{\theta} \\
& +\sum_{\theta}\left[\left(\frac{2(1+\cos \theta)(3-\cos \theta)^{-1 / 2} \sqrt{2}}{1+\sqrt{2}(1-\cos \theta)^{1 / 2}(3-\cos \theta)^{-1 / 2}}-4\right) \theta^{-1}\right] .
\end{aligned}
$$

Since the limit of the function in the brackets as $\theta \downarrow 0$ is $-2 \sqrt{2}$, there exists a constant $C_{12} \in(0, \infty)$ such that for all $N \in \mathbb{N}$,

$$
\left|\sum_{\theta}\left[\left(\frac{2(1+\cos \theta)(3-\cos \theta)^{-1 / 2} \sqrt{2}}{1+\sqrt{2}(1-\cos \theta)^{1 / 2}(3-\cos \theta)^{-1 / 2}}-4\right) \theta^{-1}\right]\right| \leq C_{12} N .
$$

Combining (2.54)-(2.60), we get

$$
\left|-\sum_{\theta} f_{\theta}^{\prime}\right|_{\beta=\beta_{c}}-\sum_{\theta} \frac{4}{\theta} \mid \leq\left(C_{11}+C_{12}\right) N+8 C_{9} N \ln \ln N .
$$

By using (1.8) and noting that $\theta=(2 n-1) \pi /(2 N)$, we have

$$
\lim _{N \rightarrow \infty} \frac{-t}{\sqrt{4 M N \ln N}}\left[\left.\sum_{\theta} f_{\theta}^{\prime}\right|_{\beta=\beta_{c}}+\frac{8 N}{\pi} \sum_{n=1}^{N} \frac{1}{2 n-1}\right]=0 .
$$

This completes the proof of the lemma by applying Lemma 2.7 .

Our last lemma is about the asymptotic behavior of the second term on the RHS of (2.52). 
Lemma 2.11. For any $\tilde{\beta} \in\left(\beta_{c}-t / \sqrt{4 M N \ln N}, \beta_{c}\right)$, we have

$$
\left.\lim _{N \rightarrow \infty} \frac{t^{2}}{8 M N \ln N} \sum_{\theta} f_{\theta}^{\prime \prime}\right|_{\beta=\tilde{\beta}}=0 .
$$

Proof: Since $g_{\theta} \geq 0$ for each $\beta \in\left(0, \beta_{c}\right]$, we have for each $\beta \in\left(0, \beta_{c}\right]$ and $\theta \in(0, \pi]$,

$$
\left|1+e^{-4 M \gamma_{\theta}}+\left(1-e^{-4 M \gamma_{\theta}}\right) g_{\theta}\right| \geq 1 \text {. }
$$

Applying this to (2.41), we obtain for each $\beta \in\left(0, \beta_{c}\right]$ and $\theta \in(0, \pi]$,

$$
\begin{aligned}
\left|f_{\theta}^{\prime \prime}\right| \leq & 4 M\left|g_{\theta}-1\right|\left|\gamma_{\theta}^{\prime \prime}\right| e^{-4 M \gamma_{\theta}}+16 M^{2}\left|g_{\theta}-1\right|\left|\gamma_{\theta}^{\prime}\right|^{2}+8 M\left|\gamma_{\theta}^{\prime}\right|\left|g_{\theta}^{\prime}\right| e^{-4 M \gamma_{\theta}}+\left|g_{\theta}^{\prime \prime}\right| \\
& +16 M^{2}\left|g_{\theta}-1\right|^{2}\left|\gamma_{\theta}^{\prime}\right|^{2}+\left|g_{\theta}^{\prime}\right|^{2}+8 M\left|\gamma_{\theta}^{\prime}\right|\left|g_{\theta}-1\right|\left|g_{\theta}^{\prime}\right| e^{-4 M \gamma_{\theta}}
\end{aligned}
$$

where $g_{\theta}^{\prime}$ and $g_{\theta}^{\prime \prime}$ are computed in (2.42) and (2.43). By (2.3), (2.32), and Lemmas 2.3 and 2.6, one has for all large $N$, all $\beta \in\left(\beta_{c}-1 / \sqrt{4 M N \ln N}\right.$, $\left.\beta_{c}+1 / \sqrt{4 M N \ln N}\right)$ and all $\theta \in(0, \pi]$,

$$
\begin{aligned}
& \left|g_{\theta}\right|=\cosh (2 \beta)\left|[\operatorname{csch}(2 \beta)-\cos \theta] \operatorname{csch}\left(\gamma_{\theta}\right)\right| \leq 6, \\
& \left|\gamma_{\theta}^{\prime}\right|=2 \cosh (2 \beta)|1+\operatorname{csch}(2 \beta)|\left|[1-\operatorname{csch}(2 \beta)] \operatorname{csch}\left(\gamma_{\theta}\right)\right| \leq 10 .
\end{aligned}
$$

By (2.32), (2.33), (2.42), (2.43), and Lemmas 2.3 and 2.6, there exist constants $C_{13}, \ldots, C_{18} \in(0, \infty)$ such that for all large $N$, all $\beta \in\left(\beta_{c}-1 / \sqrt{4 M N \ln N}\right.$, $\left.\beta_{c}+1 / \sqrt{4 M N \ln N}\right)$ and all $\theta \in(0, \pi]$,

$$
\begin{array}{ll}
\left|\gamma_{\theta}^{\prime}\right| \leq \frac{C_{13}}{\sqrt{4 M N \ln N}} \operatorname{csch}\left(\gamma_{\theta}\right), & \left|\gamma_{\theta}^{\prime \prime}\right| \leq C_{14} \operatorname{csch}\left(\gamma_{\theta}\right), \\
\left|g_{\theta}^{\prime}\right| \leq C_{15} \operatorname{csch}\left(\gamma_{\theta}\right), & \left|g_{\theta}^{\prime \prime}\right| \leq C_{16}+C_{17} \operatorname{csch}\left(\gamma_{\theta}\right)+C_{18} \operatorname{csch}^{2}\left(\gamma_{\theta}\right) .
\end{array}
$$

Combining (2.63)-(2.67), we get that there exist constants $C_{19}, C_{20}, C_{21} \in(0, \infty)$ such that

$$
\begin{aligned}
\left|f_{\theta}^{\prime \prime}\right| \leq & C_{16}+C_{17} \operatorname{csch}\left(\gamma_{\theta}\right)+C_{19} \operatorname{csch}^{2}\left(\gamma_{\theta}\right) \\
& +C_{20} M \operatorname{csch}\left(\gamma_{\theta}\right) e^{-4 M \gamma_{\theta}}+C_{21} M^{2} \frac{\operatorname{csch}^{2}\left(\gamma_{\theta}\right)}{M N \ln N}
\end{aligned}
$$

for all large $N$, all $\beta \in\left(\beta_{c}-1 / \sqrt{4 M N \ln N}, \beta_{c}\right)$ and all $\theta \in(0, \pi]$. This, (1.8), and Lemmas 2.8 and 2.9 complete the proof of the lemma.

Remark 2.12. The only place where we actually use $t \geq 0$ in the proof of Proposition 2.5 (and thus Theorem 1.1) is (2.62). It seems possible to generalize this proof to $t \in \mathbb{R}$ by a more careful analysis of (2.41) or using $f_{\theta}^{\prime \prime \prime}$.

We have all the ingredients to prove (2.19) in Proposition 2.5.

Proof of (2.19) in Proposition 2.5: This follows from (2.52), and Lemmas 2.10 and 2.11 .

\section{Acknowledgements}

This research was partially supported by STCSM grant 17YF1413300. The author thanks Chuck Newman for many useful discussions related to this work. The author also thanks an anonymous referee for valuable comments and suggestions. 


\section{References}

Abraham, D. B. Block spins in the edge of an Ising ferromagnetic half-plane. $J$. Statist. Phys., 19, 553-556 (1978). DOI: 10.1007/BF01011699.

Billingsley, P. Probability and measure. Wiley Series in Probability and Mathematical Statistics. John Wiley \& Sons, Inc., New York, third edition (1995). ISBN 0-471-00710-2. MR1324786.

Camia, F., Garban, C., and Newman, C. M. Planar Ising magnetization field I. Uniqueness of the critical scaling limit. Ann. Probab., 43 (2), 528-571 (2015). MR3305999.

De Coninck, J. Scaling limit of the energy variable for the two-dimensional Ising ferromagnet. Comm. Math. Phys., 95 (1), 53-59 (1984). MR757054.

De Coninck, J. On limit theorems for the bivariate (magnetization, energy) variable at the critical point. Comm. Math. Phys., 109 (2), 191-205 (1987). MR880413.

De Coninck, J. and Newman, C. M. The magnetization-energy scaling limit in high dimension. J. Statist. Phys., 59 (5-6), 1451-1467 (1990). MR1063207.

Di Francesco, P., Saleur, H., and Zuber, J.-B. Critical Ising correlation functions in the plane and on the torus. Nuclear Phys. B, 290 (4), 527-581 (1987). MR922845.

Hecht, R. Correlation Functions for the Two-Dimensional Ising Model. Phys. Rev., 158, 557-561 (1967). DOI: 10.1103/PhysRev.158.557.

Hongler, C. Conformal invariance of Ising model correlations. Ph.D. thesis, Univ. Geneva (2010).

Hongler, C. and Smirnov, S. The energy density in the planar Ising model. Acta Math., 211 (2), 191-225 (2013). MR3143889.

McCoy, B. M. and Wu, T. T. The two-dimensional Ising model. Harvard University Press, Cambridge, MA (1973). ISBN 0-674-91440-6. MR3618829.

Newman, C. M. A general central limit theorem for FKG systems. Comm. Math. Phys., 91 (1), 75-80 (1983). MR719811. 